\title{
In-vivo EEG Changes During A Panic Attack in A Patient With Specific Phobia
}

\author{
Hessel J. Engelbregt ${ }^{\mathrm{a}, \mathrm{d}}$, Daniel Keeser ${ }^{\mathrm{b}}$, Vincent H.L. Promes ${ }^{\mathrm{a}}$, \\ Sonja Verhagen-Schouten ${ }^{\mathrm{a}}$, Jan Berend Deijen ${ }^{\mathrm{c}}$
}

\begin{abstract}
Since early adolescence a 47-year-old male suffers from specific phobia with panic disorder (PD) which is similar to agoraphobia. The panic starts when he crosses the borders of his domicile, regardless of the type of transport. On request of client and his GP, we recorded his EEG during a car trip from his house to a distant village. We aimed to explore underlying EEG changes that may accompany the initiation and the progression of his panic attacks. The panic attack took place during the car-ride where we detected an increase of frontal beta $(\beta)$ activity $(13-26 \mathrm{~Hz})$ and a relatively sudden decrease of frontal-central theta $(\theta)$ activity $(3-6 \mathrm{~Hz})$. These findings suggest that EEG recordings can be useful to monitor the beginning and the end of a panic attack and may determine the exact EEG changes that initiate a panic attack.
\end{abstract}

Keywords: Phobia; Panic disorder; Panic attack; Agoraphobia; EEG recordings; Beta activity; Theta activity

\section{Introduction}

There are relatively few publications on panic disorder (PD),

\footnotetext{
Manuscript accepted for publication October 21, 2011

${ }^{\mathrm{a}}$ Hersencentrum, Amsterdam, The Netherlands

${ }^{b}$ Department of Psychiatry and Psychotherapy and Department of Clinical Radiology, Ludwig-Maximilian University, Munich, Germany

${ }^{c}$ VU University, Department of Clinical Neuropsychology, Amsterdam, The Netherlands

${ }^{\mathrm{d}}$ Corresponding author: Hessel J. Engelbregt, Hersencentrum, Amsterdam, Marnixstraat 364-366, 1016 XW Amsterdam, The Netherlands. Email: hessel@hersencentrum.nl

doi:10.4021/jmc403e
}

with or without (agora) phobia using EEG. Gordeev described patterns and auditory event related potentials (ERP) of the EEG in the resting state condition in a group of 77 agoraphobic patients with PD [1]. The author compared these patterns and ERPs to those in a group of 28 PD patients without agoraphobia. In the agoraphobic group he found reduced P300 ERPs as well as alpha activity and an increase in beta activity in the right hemisphere. The group without agoraphobia showed an increase of theta activity in the left temporal areas. These abnormalities in the two groups with PD suggest respectively an increased activity in the ascending mesencephalic reticular formation and increased activity in the limbic structures. Lopes and colleagues induced panic attacks in a group of 15 patients with PD by a relative increase of $\mathrm{CO} 2(35 \%)$ and atmospheric compressed air during EEG recording [2]. The quantitative EEG data (qEEG) during the panic attack (12 patients actually got a PD after the $\mathrm{CO} 2$ procedure) were subtracted from the $\mathrm{qEEG}$ data in the resting state condition. During the panic attack a relative increase in beta activity was observed at the right temporal region (T4 according to international 10-20 EEG system) [3]. Further, an increase of alpha activity right frontally (F4) and a relative decrease of left temporally (T5) theta power activity was observed.

In a study on the relation between anxiety and EEG parameters in general the traditional alpha frequencies ( 7.5 to $12.5 \mathrm{~Hz})$ were divided into three subtypes $(\alpha 1, \alpha 2$ and $\alpha 3)$. Results indicated that increased anxiety was positively associated with low alpha activity and negatively with high alpha activity (up to $12.5 \mathrm{~Hz}$ ). In addition, the authors observed a negative relationship between delta and alpha 2 activity [4].

Level of anxiety may be assessed by measuring testosterone and cortisol levels. These hormones respectively reduce and enhance (conscious) fear. Testosterone is known to be the end product of the hypothalamic-pituitary gonadal (HPG) axis. Within this system, cortisol acts as antagonist. In healthy subjects a decrease of delta-beta coherence after the administration of testosterone was found [5]. Also, a positive correlation has been found between cortisol levels and delta-beta coherence [6]. Another interesting finding on delta-beta coherence was reported by Putman [7]. Participants in his study completed questionnaires relating to anxiety and 
Table 1. Results of Paired T-tests, Laplacian Electrode Montage

\begin{tabular}{|c|c|c|c|c|c|}
\hline & Delta & Theta & Alpha & Beta & High Beta \\
\hline & $1.0-4.0 \mathrm{~Hz}$ & 4.0-8.0 Hz & 8.0-12.0 Hz & $12.0-25.0 \mathrm{~Hz}$ & $25.0-30.0 \mathrm{~Hz}$ \\
\hline FP1-CSD & $0.000000 *$ & $0.000000^{*}$ & $0.000884 *$ & 0.375551 & 0.738068 \\
\hline FP2-CSD & $0.001134^{*}$ & $0.008720^{*}$ & 0.054016 & 0.505792 & 0.205574 \\
\hline F3-CSD & $0.000111^{*}$ & $0.001396^{*}$ & 0.108641 & 0.225776 & 0.084373 \\
\hline F4-CSD & $0.000111 *$ & 0.058881 & 0.257663 & 0.260596 & $0.001678^{* *}$ \\
\hline C3-CSD & $0.000000^{*}$ & $0.000000^{*}$ & 0.893378 & 0.903372 & $0.024773 * *$ \\
\hline C4-CSD & $0.001141 *$ & $0.009590 *$ & 0.399821 & 0.889049 & 0.284939 \\
\hline P3-CSD & $0.002326^{*}$ & 0.887219 & 0.066375 & 0.095668 & $0.045029 * *$ \\
\hline P4-CSD & 0.154077 & $0.049271 *$ & 0.619726 & 0.195221 & 0.636363 \\
\hline O1-CSD & 0.063548 & $0.045359 *$ & 0.726813 & $0.000000 * *$ & $0.000946^{* *}$ \\
\hline $\mathrm{O} 2-\mathrm{CSD}$ & $0.029978^{*}$ & $0.003456^{*}$ & 0.234515 & $0.000177^{* *}$ & 0.102119 \\
\hline F7-CSD & $0.000252 *$ & $0.026553^{*}$ & 0.113575 & 0.091022 & 0.068255 \\
\hline F8-CSD & $0.034135^{*}$ & $0.003653^{*}$ & $0.015471^{*}$ & 0.384530 & 0.894694 \\
\hline T3-CSD & 0.155160 & 0.442093 & 0.180945 & $0.002244 * *$ & $0.001267 * *$ \\
\hline T4-CSD & 0.060941 & 0.251863 & 0.488802 & 0.229033 & 0.586348 \\
\hline T5-CSD & 0.145868 & 0.871427 & 0.247009 & $0.000007^{* *}$ & $0.000006^{* *}$ \\
\hline T6-CSD & $0.000862 *$ & $0.001212 *$ & 0.898031 & $0.034528 * *$ & 0.428333 \\
\hline Fz-CSD & $0.005142 *$ & $0.000000 *$ & 0.274705 & 0.190335 & $0.012400^{* *}$ \\
\hline $\mathrm{Cz}-\mathrm{CSD}$ & $0.000001 *$ & $0.000000^{*}$ & 0.296640 & 0.068157 & $0.001699 * *$ \\
\hline Pz-CSD & 0.265256 & 0.079281 & 0.449113 & $0.000011^{* *}$ & 0.386167 \\
\hline
\end{tabular}

In this two-tailed test, $\alpha<0.05$ percent is considered significant. * values indicate voltage decrements in the frequency band (columns) at a specific area on the skull (rows). ${ }^{* *}$ values indicate increased voltage.

risk avoidance. Also EEG was recorded at rest. A decreased beta-delta coherence in both risk-avoiding people and anxious patients was observed. Thus, the magnitude of delta-beta coherence might be an interesting index to measure state anxiety, while a relatively low delta-beta coherence in resting EEG can be an indication of an anxious personality trait.

To elucidate underlying neurophysiological mechanisms of panic attacks we recorded EEG in a male patient during a car trip which was known to trigger a panic attack. The aim was to get more insight in the EEG changes that may contribute to the initiation of his panic attacks.

\section{Case Report}

The 47-year-old patient was referred to us by his GP. In the past, he had been diagnosed with an anxiety disorder [Specific Phobia (DSM-IV: 300.29)] with panic attacks according to DSM-IV and comorbid major depressive disorder (DSMIV: 296.20) [8]. More specifically, the panic attack was usually triggered after leaving his residence, regardless of the type of transport. For about 15 years he used lorazepam and paroxetine (standard doses). These medications were originally prescribed for agoraphobia (DSM-IV: 300.24 ) and de- 

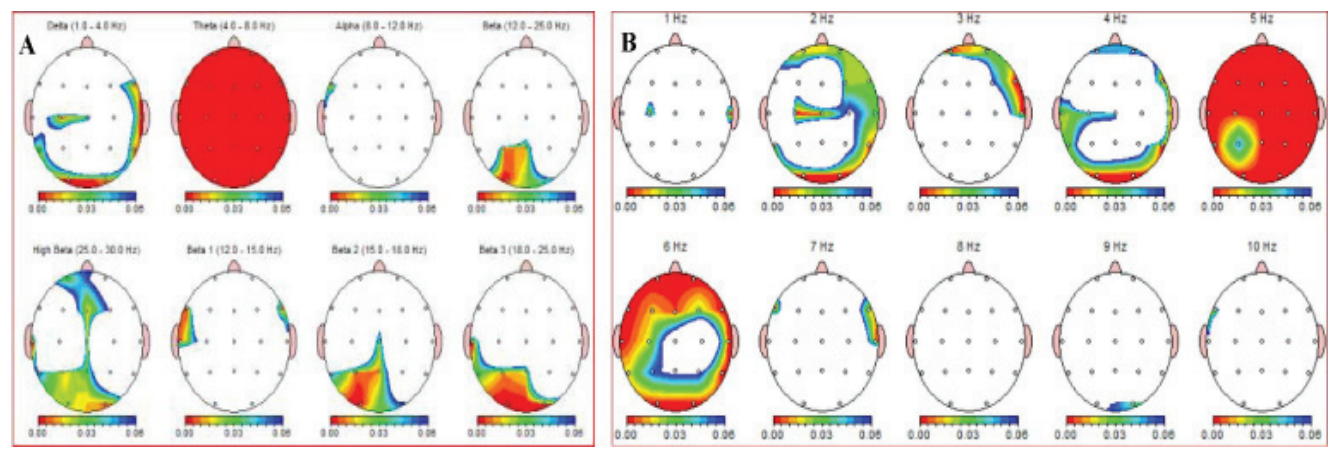

Figure 1. A (left) and B: EEG-recordings during panic attack minus pre-panic baseline EEG. The red-blue color scale represents $\mathrm{P}<0.01$ (red) and $\mathrm{P}<0.05$ (blue). The "Theta" $(4-8 \mathrm{~Hz})$ in image $\mathrm{A}$ is a representation of a significant reduction in $\theta$ activity $(P<0.01)$ across the whole surface of the skull. This Figure shows Linked Ears reference.

pression.

The reason for his referral to Hersencentrum (Brain Centre) in Amsterdam was the chronicity and worsening of patient's symptoms. We recorded resting state EEG and did not find any abnormalities in morphology of the qualitative EEG. However, examining the quantitative EEG data (using the NeuroGuide Normative Database [9]) we considered a treatment program including psychotherapy (CBT) in combination with an experimental EEG-neurofeedback trial. The EEG-neurofeedback training was aimed to reduce theta (4-7 Hz) and beta $(13-26 \mathrm{~Hz})$ activity, which were relatively high in the resting state EEG compared to a normative database. Although patient and family perceived an improvement in social interactions and a reduction of stress-related complaints during the treatment, the complaints pertaining to phobia persisted. Therefore, we decided to measure EEG during a panic attack to find evidence which could give rise for a new treatment method.

In the present study, patient and EEG assistant were sitting in the back of a comfortable car. EEG with open eyes was recorded by means of a Deymed Truscan 32-channel system in combination with a 19 channel electrode cap. Electrode skin impedance was always less than $5 \mathrm{k} \Omega$. The electrodes were placed according to the International 10-20 system. An electrode at Fpz served as ground electrode. To minimize the uncomfortable setting for the patient, electrooculography (EOG) was omitted. The EEG system was connected to a portable computer.

The trip took him beyond the borders of his hometown, which pertains to patient's specific phobia. We expected that a panic attack would occur during the ride while EEG was being recorded. Each minute, the patient marked the severity of his anxiety on a $0-10$ scale.

The patient's scores on the anxiety scale were marked on the digital EEG. Tags were: increased fear, anxiety, start of panic attack and stop car.

In order to maximize safety during the ride, we agreed with the patient a maximum anxiety rate of 8 before stopping the car. After approximately one kilometer beyond the borders of his home (a journey of about 15 minutes), the subject reported a panic score of 8 . The driver stopped the car and after several minutes the EEG equipment was stored and the homeward journey was initiated.

EEG was analyzed by means of the program Insight II (Version 2004.11.22, Persyst Development Corporation, Prescott, Arizona) for qualitative analysis and automatic

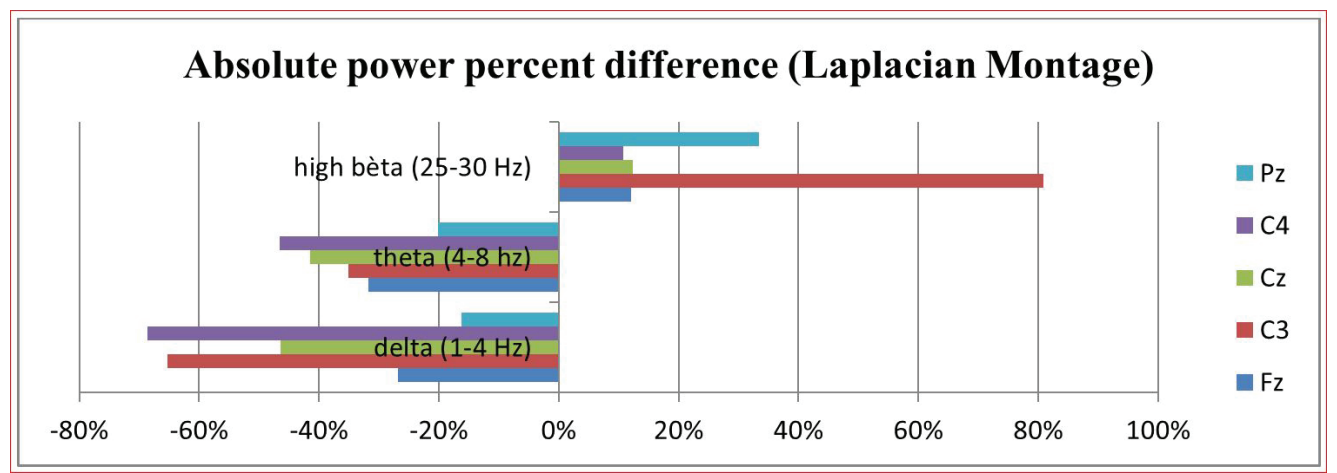

Figure 2. Result of absolute power percentage difference between pre-panic and panic state of delta, theta and high beta frequencies at central locations, Laplacian montage. 
screening of epileptic phenomena. Prior to quantitative analysis, the EEG recording was screened by a neurologist for seizure activity and/or abnormal EEG patterns. There were no signs of either. Data selection for quantitative EEG analysis was done by a neuropsychologist. These selections were verified by an independent EEG expert. For quantitative analysis NeuroGuide (Version 2.6.6) was used. The entire recording lasted 27.5 minutes. Due to artefacts in the EEG (primarily muscle tension), the total time of EEG samples (artefact free selections of the original recording) which were used for quantitative analysis was 1:28 minutes during the panic attack and 2:43 minutes prior to the attack. Both Linked-Ears (LE, for general indication of activity) and Laplacian (for indication of source of activity) montage were used for comparison of both conditions.

Individual transformed EEG selections of period 1 (p1, period before the panic attack, with an EEG sample of 2:43 minutes) and period 2 (p2, during the panic attack, with an EEG sample of 1:28 minutes.) were compared by paired ttests using NeuroGuide 2.6.6 Normative Database. Power changes of individual EEG frequencies between 0 and $50 \mathrm{~Hz}$ of p1 minus p 2 were calculated. Table 1 shows the outcome of the t-tests (P-value). In this two-tailed test, $\alpha$ below 5 percent is considered significant. We also looked at the duration of cross-spectral phase lock between the delta (1-4 Hz) and beta frequencies. Phase locking is consistent firing of a group of cells. When multiple groups of cells fire in phase, the occurrence of communication between these groups of neurons can be assumed [10]. Phase lock is a similar principle as consistency and correlation between the two measures is high [11]. Beta frequencies above were divided into four subtypes, $12-15 \mathrm{~Hz}, 15-18 \mathrm{~Hz}, 18-25 \mathrm{~Hz}$ and $25-30 \mathrm{~Hz}$. We looked at the following frequency bands; $\delta(1-4 \mathrm{~Hz}), \Theta(4-8$ $\mathrm{Hz}), \alpha(8-12 \mathrm{~Hz}), \beta(12-15 \mathrm{~Hz}), \beta 2$ (15-18 Hz), $\beta 3$ (18-25 $\mathrm{Hz})$ and $\beta 4(25-30 \mathrm{~Hz})$. Results are given in power.

With respect to EEG changes during the panic attack, the power difference between $\mathrm{p} 1$ and $\mathrm{p} 2$ is summarized in Table 1. This Table shows the power of the following frequency bands; $\delta$ (1-4 Hz), $\theta$ (4-8 Hz), $\alpha$ (8-12 Hz), $\beta$ (12-15 $\mathrm{Hz}), \beta 2(15-18 \mathrm{~Hz}), \beta 3(18-25 \mathrm{~Hz})$ and $\beta 4(25-30 \mathrm{~Hz})$. Figure 1 visualizes the significance of changes between panic and pre-panic voltage of EEG frequencies. The most striking difference is a significant drop in theta activity. The right part of the figure shows that this is specifically a reduction of 5 and $6 \mathrm{~Hz}$ activity in this case.

Also, widely spread across the cortex, a significant decrease in $\delta$ activity was present during the panic attack. Finally, a significant increase in $\beta$ activity, especially in more posterior regions, is seen. Figure 2 shows absolute power percent differences of $\delta, \theta$ and $\beta 3$ activity at five central locations. The overall image of Linked Ears (LE) and Laplacian montages is equal. In the present study there were no significant differences between the duration of the $\delta$ - $\beta$ phase lock during and prior to the panic attack.

\section{Discussion}

The aim of this study was to examine changes in EEG between rest condition and during a panic attack in a patient suffering from specific phobia which is similar to agoraphobia. Based on the anamnesis, it could be assumed that the protocol would provoke a panic attack. An ethical question that arises is whether it is justified, or at least in the interest of the patient, to force a panic attack.

Within the research team there were doubts about both need and usefulness of this exposure. The decision to agree with the request of patient and his family was taken after extensive study of the previous treatments (15 years of medication and numerous therapies) and after a half year of intense monitoring of the patient during combined psychotherapy and EEG-neurofeedback. Although patient and his family reported some improvement in his daily functioning and mood state, there was no difference in the severity of his primary complaint, the phobia. On forehand, patient described the regular process towards and during his panic attacks in detail. Based on his experiences, we felt that the health of the patient and the safety of all occupants in the vehicle during the recording would not be violated. Regarding the possibility of any therapy to be given on the basis of EEG findings (e.g. medication or continuation of EEG neurofeedback) information of EEG changes during his panic attacks justified the exposure in our opinion and were in consent with the patient's wishes.

The present study shows that changes occur in the EEG of a patient with panic attacks with Specific Phobia during the panic attack. Especially beta activity in the frequencies of $13-26 \mathrm{~Hz}$ in the central part of the frontal cortex increased. This increase was accompanied by a significant reduction in theta activity over the entire cortex. These findings are consistent with those of previous studies, which reported an increase in theta activity in patients with anxiety disorders while experiencing feelings of relaxation [12] In simultaneous EEG-fMRI studies slow EEG patterns were found to correlate negatively with functional connectivity in the default mode network (DMN) during resting state [13]. Reduced delta and increased beta power was positively correlated to increased functional connectivity [14]. We suggest that panic induced changes in functional connectivity patterns as found previously on resting state fMRI [15] in areas as the frontal brain, the temporal and parietal cortex and subcortical regions.

As we also recorded patient's resting EEG prior and after the current experiment, we knew that the 13-26 Hz beta and theta activity were relatively high before the panic attack compared to a normative EEG database. Therefore we hypothesize that EEG could be indicative of the risk of a panic attack at some point in time. A person with an EEG pattern that matches the expected image during a panic attack may need fewer stressors to force a panic attack. Fur- 
ther, it may be of interest to record the EEG of a large group of patients with specific phobia or agoraphobia to determine the presence of a phenotypically EEG image. If so, phenotypic images can be used to better understand therapeutic approaches useful in agoraphobia and measuring treatment effect. Finally, since there are relatively few therapies which reduce deviations in EEG, we unsuccessfully treated the patient with EEG-neurofeedback (frequency training) prior to the start of the present study. The aim of the training was to reduce patient's abnormal high beta and theta activity as was indicated by deviations from the normative database. Thus, as the relatively high theta activity in the resting-state EEG was followed by a major reduction in theta activity during the panic attack, it may be possible that reducing resting state theta activity can be useful in preventing a panic attack. It is interesting to further explore whether high theta activity in resting-state EEG increases the risk of a panic attack and/ or that the reduction of theta activity is a factor in itself for experiencing panic. Neurotherapy protocols may be adapted after obtaining evidence on this issue.

\section{Conflicts of Interest}

The authors declare that they have no conflict of interests.

\section{References}

1. Gordeev SA. Clinical and psychophysiological study of patients with panic attacks with or without agoraphobic disorders. Zh Nevrol Psikhiatr Im S S Korsakova. 2007;107(4):54-58.

2. Lopes FL, Oliveira MM, Freire RC, Caldirola D, Perna G, Bellodi L, Valenca AM, et al. Carbon dioxide-induced panic attacks and quantitative electroencephalogram in panic disorder patients. World J Biol Psychiatry. 2010;11(2 Pt 2):357-363.

3. Jaspers HH. The ten twenty electrode system of the international federation. Electroencephalogr. Clin Neurophysiol. 1958;10:371-375.

4. Knyazev GG, Savostyanov AN, Levin EA. Alpha oscillations as a correlate of trait anxiety. Int J Psychophysi- ol. 2004;53(2):147-160.

5. Schutter DJ, van Honk J. Decoupling of midfrontal delta-beta oscillations after testosterone administration. Int J Psychophysiol. 2004;53(1):71-73.

6. Schutter DJ, van Honk J. Salivary cortisol levels and the coupling of midfrontal delta-beta oscillations. Int J Psychophysiol. 2005;55(1):127-129.

7. Putman P. Resting state EEG delta-beta coherence in relation to anxiety, behavioral inhibition, and selective attentional processing of threatening stimuli. Int J Psychophysiol. 2011;80(1):63-68.

8. American Psychiatric Association (1994). Diagnostic and Statistical Manual of Mental Disorders (4th ed.) Washington, DC: American Psychiatric Association Press.

9. Thatcher RW Normative EEG databases and EEG biofeedback. J Neurother. 1998;2 (4):8-39.

10. Womelsdorf T, Schoffelen JM, Oostenveld R, Singer W, Desimone R, Engel AK, Fries P. Modulation of neuronal interactions through neuronal synchronization. Science. 2007;316(5831):1609-1612.

11. Thatcher RW, North DM, Biver CJ. Intelligence and EEG phase reset: a two compartmental model of phase shift and lock. Neuroimage. 2008;42(4):1639-1653.

12. Suetsugi M, Mizuki Y, Ushijima I, Kobayashi T, Tsuchiya K, Aoki T, Watanabe Y. Appearance of frontal midline theta activity in patients with generalized anxiety disorder. Neuropsychobiology. 2000;41(2):108-112.

13. Scheeringa R, Bastiaansen MC, Petersson KM, Oostenveld R, Norris DG, Hagoort P. Frontal theta EEG activity correlates negatively with the default mode network in resting state. Int J Psychophysiol. 2008;67(3):242251.

14. Hlinka J, Alexakis C, Diukova A, Liddle PF, Auer DP. Slow EEG pattern predicts reduced intrinsic functional connectivity in the default mode network: an inter-subject analysis. Neuroimage. 2010;53(1):239-246.

15. Dieler AC, Samann PG, Leicht G, Eser D, Kirsch V, Baghai TC, Karch S, et al. Independent component analysis applied to pharmacological magnetic resonance imaging (phMRI): new insights into the functional networks underlying panic attacks as induced by CCK-4. Curr Pharm Des. 2008;14(33):3492-3507. 